\title{
Procedural Precautions and Personal Protective Equipment during Head and Neck Instrumentation in the COVID-19 Era
}

\author{
Jason Y K Chan, FRCSEd (ORL) ${ }^{1}$ \\ ${ }^{1}$ The Chinese University of Hong Kong
}

April 27, 2020

\begin{abstract}
Background: Otolaryngologists represent a subset of healthcare workers uniquely vulnerable to COVID-19 transmission. Given the segmentation of extant guidelines concerning precautions and protective equipment for SARS-CoV2, we aimed to provide consolidated recommendations regarding appropriate personal protective equipment (PPE) in head neck surgery during the COVID-19 era.

Methods: Guidelines published by international and United States governing bodies were reviewed in conjunction with published literature concerning COVID-19 transmission risk, testing, and PPE, to compile situation-specific recommendations for head and neck providers managing COVID-19 patients.

Results: High-quality data regarding the aerosolization potential of head and neck instrumentation and appropriate PPE during head and neck surgeries are lacking. However, extrapolation of recommendations by governing bodies suggest strongly that head and neck mucosal instrumentation warrants strict adherence to airborne-level precautions.

Conclusion: We present a series of situation-specific recommendations for PPE use and other procedural precautions for otolaryngology providers to consider in the COVID-19 era.
\end{abstract}

\section{Bharat A. Panuganti, $\mathrm{MD}^{1^{*}}$; John Pang, $\mathrm{MD}^{1^{*}}$; Joseph Califano, MD ${ }^{1}$; Jason Y K Chan, FRCSEd (ORL) ${ }^{2}$.}

${ }^{1}$ University of California-San Diego, Department of Surgery, Division of Otolaryngology-Head and Neck Surgery, San Diego, CA, USA.

${ }^{2}$ The Chinese University of Hong Kong, Department of Otorhinolaryngology, Head and Neck Surgery, Shatin, Hong Kong.

Bharat A. Panuganti and John Pang are co-first authors.

\section{Corresponding Authors:}

\section{Jason Y K Chan, FRCSEd (ORL)}

${ }^{2}$ The Chinese University of Hong Kong, Department of Otorhinolaryngology, Head and Neck Surgery, Shatin, Hong Kong

E-Mail: jasonchan@ent.cuhk.edu.hk

\section{Joseph Califano}

Division of Otolaryngology-Head and Neck Surgery, Department of Surgery, University of

California-San Diego 
3855 Health Sciences Dr, MC 0803, La Jolla, CA 92037-0803, USA.

Email: jcalifano@ucsd.edu

Running Title: Precautions and PPE in Head and Neck Surgery during COVID-19

Keywords: COVID-19, personal protective equipment, high-risk procedures, perioperative protocols

\section{Abstract}

Background: Otolaryngologists represent a subset of healthcare workers uniquely vulnerable to COVID-19 transmission. Given the segmentation of extant guidelines concerning precautions and protective equipment for SARS-CoV2, we aimed to provide consolidated recommendations regarding appropriate personal protective equipment (PPE) in head neck surgery during the COVID-19 era.

Methods: Guidelines published by international and United States governing bodies were reviewed in conjunction with published literature concerning COVID-19 transmission risk, testing, and PPE, to compile situation-specific recommendations for head and neck providers managing COVID-19 patients.

Results: High-quality data regarding the aerosolization potential of head and neck instrumentation and appropriate PPE during head and neck surgeries are lacking. However, extrapolation of recommendations by governing bodies suggest strongly that head and neck mucosal instrumentation warrants strict adherence to airborne-level precautions.

Conclusion: We present a series of situation-specific recommendations for PPE use and other procedural precautions for otolaryngology providers to consider in the COVID-19 era.

rocedural Precautions and Personal Protective Equipment during Head and Neck Instrumentation in the COVID-19 Era

\section{Introduction}

The COVID-19 pandemic has afflicted more than 1.8 million individuals in over 183 countries and territories, prompting widespread debate about the mechanisms of disease transmission, personal protection against spread, and treatment methods. ${ }^{1}$ Healthcare workers represent a uniquely vulnerable population, given their close proximity to infected patients in settings with abundant fomites, with further increased risk in the setting of aerosol-generating procedures (AGP). Scope of practice in otolaryngology necessitates instrumentation of the upper aerodigestive tract, wherein significant viral loads are found in both symptomatic and asymptomatic patients. As such, in the absence of widespread screening, otolaryngologists are at heightened risk of SARS-CoV2 contraction in common practice. For healthcare workers, the use of adequate personal protective equipment (PPE) is crucial for the protection of both patients and providers. In fact, during the SARS-CoV1 epidemic, healthcare workers who used PPE inconsistently were more likely to develop SARS-CoV1. ${ }^{2}$ In light of the widespread shortage of PPE and the high, relative risk of transmission during head and neck procedures (via mucus, blood, or aerosolized virions), we present situation-specific guidelines for PPE use by otolaryngologists during the COVID-19 pandemic, based on our compilation and collective interpretation of existing recommendations and data. ${ }^{3}$

\section{Overview of Method of Transmission/PPE}

The World Health Organization recognizes COVID-19 as a droplet-borne disease unless an aerosol-generating procedure is performed (e.g. non-invasive positive pressure ventilation, bronchoscopy, open suctioning, endotracheal intubation, cardiopulmonary resuscitation, tracheostomy, and administration of nebulized treatment). Currently, coughing and sneezing are categorized as droplet-producing, not aerosolizing, by both the Centers for Disease Control (CDC) and World Health Organization (WHO). Droplet transmission occurs via respiratory droplets between 5-10 micrometers when an at-risk individual is within 1 meter of the source. Airborne transmission occurs in so-called droplet "nuclei" measuring fewer than 5 micrometers capable of traveling for distances greater than 1 meter, such as the case during AGPs. While several publications have reported SARS-CoV2 aerosolization risk with coughing or sneezing, although, so far, the WHO has 
maintained that additional research is necessary to verify these claims, citing issues either with using RNA detection as a proxy for the presence of viable virus, or the method by which inoculum was aerosolized. ${ }^{4-6}$

Loose-fitting, standard surgical facemasks, together with a face-shield or eye protection, are considered protective against droplets. Fit-tested N95 filtering facepiece respirators, along with tight-fitting eye protection or face-shields with side protection to shield the conjunctiva are indicated for providers when there is a risk for airborne transmission. Powered air-purifying respirators (PAPR) represent a reusable alternative to N95s for protection against airborne transmission. Although there is a quantitative difference in the filtering efficacy of N95 filtration masks (assigned protection factor [APF] of 10, indicating that $90 \%$ of airborne particles are blocked from inhalation) versus PAPR (APF of up to 1000 for a full facepiece PAPR), neither the CDC nor the WHO have clearly characterized a clinically-discernible difference with standard use. ${ }^{7}$ While the N95 requires mask-fit testing, PAPR requires training to guide proper assembly and troubleshooting of components (e.g. battery, hose, filter, etc.), donning and doffing, and may pose unique visibility issues (e.g. fogging, inability to use headlight) in the operating room. Moreover, headaches are common amongst healthcare workers wearing N95 masks ${ }^{8}$, a phenomenon postulated to be related to impaired gas exchange with retention of carbon dioxide, or mechanical factors associated with prolonged N95 mask usage. ${ }^{9}$

\section{Testing}

History and physical exam alone are often insufficient to guide PPE and procedural decision-making, as up to $80 \%$ of patients may be mildly symptomatic or asymptomatic COVID-19 carriers. ${ }^{1011}$ Clear protocols to advise testing, with accommodations for limitations in resources, are therefore important to appropriately protect providers and patients against transmission.

As it stands, there is no "gold standard test"; negative results do not preclude SARS-CoV2 infection and should not be used as the sole basis for patient management decisions (West CP). Rather, symptoms, history, and test findings should be used together to guide assessment of COVID-19 status. ${ }^{12}$ In otolaryngology, COVID-19 status (known or inferred) is integral to direct appropriate PPE for all involved staff in the operating room or clinic setting. Currently, the most available molecular diagnostic tests are real-time reverse-transcription polymerase chain reaction assays for two or more viral targets. However, as there are concerns for the low sensitivity and negative predictive value of a single test, a combination of two PCR tests or the addition of chest $\mathrm{CT}$ to the diagnostic algorithm have been suggested to improve sensitivity, although this combination may not be universally feasible in the preoperative setting. ${ }^{13}$ The current negative predictive value of each test is variable, but guidance for de-isolation of infected patient includes at least two upper respiratory tract samples negative for SARS-CoV-2 collected at least 24 hours apart by the European Centre for Disease Prevention and Control and the Department of Health of Hong Kong. This testing approach may potentially be used as testing kit availability and result turnaround times improve to screen patients for elective surgery. ${ }^{14}$

\section{PPE for Airway Procedures}

Otolaryngologists are commonly involved in AGPs requiring open or endoscopic airway instrumentation, including tracheostomy, direct laryngoscopy with interventions, and endotracheal intubation. A systematic review meant to assess the relative risk of transmission of SARS-CoV1 during a spectrum of AGPs found endotracheal intubation (odds ratio $[\mathrm{OR}]=6.6)$ and tracheostomy $(\mathrm{OR}=4.2)$ to be among the highest-risk procedures. ${ }^{15}$ On April 2, the American Academy of Otolaryngology (AAO) released an updated position statement recommending against elective tracheostomy within the first 2-3 weeks following intubation, in patients with high-pressure ventilatory requirements, and in patients without COVID-19 testing. ${ }^{16}$ Moreover, there is little existing evidence suggesting a definitive overall survival benefit associated with early tracheostomy among critically-ill patients. ${ }^{17}$ As such, the theoretical remaining benefits of early, elective tracheostomy (e.g. laryngotracheal morbidity) must be carefully weighed against the known risk of SARS-CoV2 transmission during tracheotomy procedures, when the option to bide time until viral clearance or ventilatory independence occurs. However, if patient circumstances (e.g. bulky head and neck tumor) necessitate a tracheostomy or elective tracheostomy is performed, appropriate PPE is strictly necessary. 
PPE for tracheostomy procedures should abide by airborne-level precautions, which include an N95 mask with eye protection or PAPR, surgical cap, long-sleeved waterproof surgical gowns, gloves, and shoe/leg covers to prevent tracking. Some groups, cultivating their experience from treatment of either SARS-CoV1 or SARSCoV2 patients, have advocated for enhanced PPE during high-risk, airway procedures, such as tracheostomy, including full face-shields or helmets with full face coverage and double gloves, or concomitant N95 and PAPR use. ${ }^{18,19}$ Other important considerations include 1) possibility of performing open tracheostomy in a negative-pressure room in the intensive care unit, to minimize unnecessary ventilatory circuit manipulation for patient transport; 2) complete muscular paralysis, when permitted (i.e. not awake tracheostomy), during the procedure to minimize coughing with an open airway; 3) minimization of electrocautery to mitigate the potential transmission of aerosolized virions through smoke ${ }^{20}$, and, 4) use of a closed tracheal suction system with a viral filter. While percutaneous tracheostomy can be considered as an alternative to intraoperative open tracheostomy, there may be similar, if not more significant, risk of aerosolization given the need for bronchoscopy and serial instrumentation of the airway for dilation. ${ }^{21}$ If awake tracheostomy cannot be avoided, additional unique considerations include adequate local anesthesia to prevent patient movement during the procedure, and a patient surgical facemask, with low-flow oxygen administration via nasal cannula, if necessary, to reduce large droplet transmission with coughing. Routine tracheostomy tube exchanges should be delayed in COVID-19 patients to limit airway manipulation; instead, simple cuff deflation should be considered to reduce mucosal irritation and facilitate phonation. If exchange is performed, standard airborne-level PPE (N95, eye protection or full faceshields, surgical gown, and gloves) should be donned by participating providers, including nurses and respiratory therapists.

Though specific guidelines have not been formulated for suspension microlaryngoscopy, the procedure exposes the distal airway with positive pressure ongoing (albeit with a seal created by the endotracheal tube cuff), and involves instrumentation of the respiratory mucosa. Given the CDC's current characterization of intubation and bronchoscopy as "high-risk" procedures (i.e. either aerosolizing or associated with poor control of respiratory secretions), airborne precautions should be strongly considered for suspension microlaryngoscopy if surgery cannot be delayed (e.g. laryngeal cancer with marginal endoscopic resectability). ${ }^{22}$ Use of laser is common during microlaryngoscopic procedures; the risk of human papilloma virus (HPV) transmission has been studied in this setting. A study by Kunachack et al. found that no viable HPV virions could be cultured from CO2 laser plume. ${ }^{23}$ Another study by Ferenczy et al. found HPV DNA in the prefilter section of the smoke evacuator used to clear the surgical field of the laser plume; the inner, postfilter sections of the smoke evacuator, however were devoid of viral DNA. ${ }^{24}$ While iatrogenic inoculation of the surgeon is not a widely reported phenomenon in the treatment of HPV related disease, it is unclear if empirical measures, including use of a smoke evacuator with a high-efficiency filter, or prior research concerning HPV would be applicable to SARS-CoV2. Lastly, jet ventilation should be avoided, if possible, given the procedure's high applied pressure without an inflated cuff as a barrier to aerosolized virions. (Table 1)

Unless the otolaryngologist is required in the operating room during endotracheal intubation of COVIDpositive or suspicious patients (e.g. if the risk of a surgical airway is high), only anesthesia providers should be present during intubation with appropriate PPE donned. A statement issued by the American Society of Anesthesiologists recommended standard airborne-level PPE (N95 respiratory with eye protection or facial protection covering the sides of the face versus PAPR, waterproof gown, and gloves) be worn by providers present during intubations for patients with confirmed or suspected COVID- $19{ }^{25}$ Additional recommendations for the intubating provider include use of videolaryngoscopy to allow for greater distance between the provider and the patient and prompt inflation of the endotracheal tube cuff after passage through the vocal cords to create a seal before initiation of positive pressure ventilation.

\section{PPE for Endoscopic Sinus or Skull Base Surgery}

The nasopharynx and nasal cavity are known to harbor significant viral loads, and in concentrations potentially proportionate to the severity of COVID-19 symptoms. ${ }^{26}$ In a commonly cited case, a patient with either no symptoms or mild flu symptoms in Wuhan underwent a trans-sphenoidal pituitary surgery in January 2020; 14 healthcare workers who were involved in the patient's care (either in the operating room or 
outside of it) were reported to have tested positive for COVID-19. The precise circumstances surrounding exposure of the 14 healthcare workers, however, are uncertain, and their COVID-19 positivity may or may not have been due to their interaction with the patient who underwent the trans-sphenoidal procedure. The CDC currently characterizes open suctioning in a COVID-19 positive patient as an AGP; as such, endoscopic transnasal procedures (e.g. endoscopic sinus surgery), with suctioning and concomitant mucosal instrumentation in a florid field, should warrant at least standard airborne-level PPE. In fact, the CDC recommends an N95 or higher-level respiratory for providers obtaining nasopharyngeal swabs in COVID-19-suspicious patients, resources permitting, reflecting the greater relative degree of caution the CDC exercises with nasal or nasopharyngeal instrumentation. ${ }^{22}$ (Table 1 )

\section{PPE for surgery of the neck without airway or mucosal exposure}

Although surgical procedures including cold steel incision and dissection, suction, and cautery can theoretically aerosolize blood and tissue particles ${ }^{27}$, the potential for SARS-CoV2 virus aerosolization appears to be minimal outside the setting of the upper aerodigestive tract. In fact, COVID-positive patients demonstrated no detectable serum SARS-CoV2 RNA, compared to sputum analysis which produced $7 \times 10^{6}-2.35 \mathrm{x}$ $10^{9}$ copies $/ \mathrm{ml}^{28}$. Therefore, the extant literature suggests that standard surgical precautions are sufficient for surgery involving the neck without mucosal membrane exposure in COVID-positive patients. Accordingly, it may be recommended that providers exposed to the surgical field (surgeons, surgical assistants, and surgical technologists) wear standard PPE for surgeries (e.g. neck dissections) without airway or mucosal exposure (i.e. loose-fitting surgical mask, gloves, eye shield, and surgical gowns). (Table 1)

\section{PPE for Otologic Surgery}

Data concerning viral burden in the middle ear and mastoid cavity is lacking, though historical studies have found coronavirus RNA in middle ear fluid. ${ }^{29}$ Recommendations have been made for enhanced eye (i.e. tight-fitting goggles or PAPR) protection when drilling is performed, which have been related to reports of transcorneal penetration of bone dust during mastoidectomy demonstrated in an experimental setting ${ }^{30}$, and the distinct uncertainty surrounding, but persistent suspicion of, viral aerosolization with bone drilling. In light of the available data, at least standard airborne precautions should be followed on COVID-positive or suspicious patients undergoing otologic surgery.

\section{Outpatient clinic}

The use of PPE for COVID-positive or suspected patients in the outpatient setting may be dictated by the nature of the patient interaction. As previously noted, the CDC advises airborne-level precautions (i.e. N95 or higher-level respirator, eye protection, gloves, and gown), and consideration of negative-pressure room, when performing aerosol-generating procedures. ${ }^{7}$ Some in vitro evidence has been interpreted to suggest that flexible nasal endoscopy, for example, while not intrinsically an AGP, may lead to aerosol generation via coughing or sneezing. ${ }^{31}$ As previously mentioned, however, the WHO classifies coughing and sneezing as droplet-generating, not aerosolizing, events. However, nasal endoscopy with suctioning would be classified as an AGP by the CDC, warranting airborne-level precautions. ${ }^{22}$ Standard head and neck physical examinations of putative or positive COVID-19 patients require droplet-level precautions, ensuring appropriate eye protection, given the need for close inspection of the nasal and/or oral cavities and potential proximity to droplet transmission with expectoration. (Table 1)

\section{Conclusion}

Despite the persistent uncertainty surrounding the anticipated trajectory of COVID-19, and the precise risk profile associated with head and neck procedures, it behooves otolaryngologists to practice responsible PPE use and abide by procedural precautions (e.g. active preoperative testing, delaying non-emergent cases) for the safety of both providers and patients. Herein, we present recommendations for otolaryngologists practicing in both the outpatient and inpatient settings that are summarized in table 1 . While the medical community's understanding of SARS-CoV2 continues to evolve, the consistent observation of safety measures is critical to limit propagation of COVID-19 in the healthcare setting. 


\section{References}

1. COVID-19 Map. Johns Hopkins Coronavirus Resource Center. https://coronavirus.jhu.edu/map.html. Accessed April 14, 2020.

2. Lau JTF, Fung KS, Wong TW, et al. SARS Transmission among Hospital Workers in Hong Kong. Emerg Infect Dis . 2004;10(2):280-286. doi:10.3201/eid1002.030534

3. Ranney ML, Griffeth V, Jha AK. Critical Supply Shortages - The Need for Ventilators and Personal Protective Equipment during the Covid-19 Pandemic. N Engl J Med . 2020;0(0):null. doi:10.1056/NEJMp2006141

4. Bourouiba L. A Sneeze. N Engl J Med . 2016;375(8):e15. doi:10.1056/NEJMicm1501197

5. van Doremalen N, Bushmaker T, Morris DH, et al. Aerosol and Surface Stability of SARS-CoV-2 as Compared with SARS-CoV-1. N Engl J Med . 2020;0(0):null. doi:10.1056/NEJMc2004973

6. Modes of transmission of virus causing COVID-19: implications for IPC precaution recommendations. https://www.who.int/news-room/commentaries/detail/modes-of-transmission-of-virus-causing-covid19-implications-for-ipc-precaution-recommendations. Accessed April 7, 2020.

7. Understanding respiratory protection options in Healthcare: The Overlooked Elastomeric || Blogs | CDC. https://blogs.cdc.gov/niosh-science-blog/2017/07/06/elastomerics/. Accessed April 7, 2020.

8. Lim EC, Seet RC, Lee KH, Wilder-Smith EP, Chuah BY, Ong BK. Headaches and the N95 face-mask amongst healthcare providers. Acta Neurol Scand . 2006;113(3):199-202.

9. Tong PSY, Kale AS, Ng K, et al. Respiratory consequences of N95-type Mask usage in pregnant healthcare workers - a controlled clinical study. Antimicrob Resist Infect Control . 2015;4. doi:10.1186/s13756-015-0086Z

10. Q\&A: Similarities and differences - COVID-19 and influenza. https://www.who.int/news-room/q-adetail/q-a-similarities-and-differences-covid-19-and-influenza. Accessed April 15, 2020.

11. Bai Y, Yao L, Wei T, et al. Presumed Asymptomatic Carrier Transmission of COVID-19. JAMA . 2020;323(14):1406-1407. doi:10.1001/jama.2020.2565

12. Covid L. ACCELERATED EMERGENCY USE AUTHORIZATION (EUA) SUMMARY COVID-19 RT-PCR TEST (LABORATORY CORPORATION OF AMERICA). :7.

13. Ai T, Yang Z, Hou H, et al. Correlation of Chest CT and RT-PCR Testing in Coronavirus Disease 2019 (COVID-19) in China: A Report of 1014 Cases. Radiology . 2020;(Journal Article):200642. doi:10.1148/radiol.2020200642

14. European Centre for Disease Prevention and Control. Discharge criteria for confirmed COVID-19 cases - When is it safe to discharge COVID-19 cases from the hospital or end home isolation? 2020;2020(Apr 14,). https://www.ecdc.europa.eu/sites/default/files/documents/COVID-19-Discharge-criteria.pdf.

15. Tran K, Cimon K, Severn M, Pessoa-Silva CL, Conly J. Aerosol generating procedures and risk of transmission of acute respiratory infections to healthcare workers: a systematic review. PloS One . 2012;7(4):e35797. doi:10.1371/journal.pone.0035797

16. Parker N. Tracheotomy Recommendations During the COVID-19 Pandemic. American Academy of Otolaryngology-Head and Neck Surgery. https://www.entnet.org/content/tracheotomy-recommendationsduring-covid-19-pandemic. Published March 27, 2020. Accessed April 7, 2020.

17. Young D, Harrison DA, Cuthbertson BH, Rowan K. Effect of Early vs Late Tracheostomy Placement on Survival in Patients Receiving Mechanical Ventilation: The TracMan Randomized Trial. JAMA . 2013;309(20):2121-2129. doi:10.1001/jama.2013.5154 
18. Sorbello M, El-Boghdadly K, Giacinto ID, et al. The Italian coronavirus disease 2019 outbreak: recommendations from clinical practice. Anaesthesia. n/a(n/a). doi:10.1111/anae.15049

19. Chee VWT, Khoo ML-C, Lee SF, Lai YC, Chin NM. Infection Control Measures for Operative Procedures in Severe Acute Respiratory Syndrome-related Patients. Anesthesiol J Am Soc Anesthesiol . 2004;100(6):1394-1398.

20. Kwak HD, Kim S-H, Seo YS, Song K-J. Detecting hepatitis B virus in surgical smoke emitted during laparoscopic surgery. Occup Environ Med . 2016;73(12):857-863. doi:10.1136/oemed-2016-103724

21. Tay JK, Khoo ML-C, Loh WS. Surgical Considerations for Tracheostomy During the COVID-19 Pandemic: Lessons Learned From the Severe Acute Respiratory Syndrome Outbreak. JAMA Otolaryngol Neck Surg . March 2020. doi:10.1001/jamaoto.2020.0764

22. CDC. Coronavirus Disease 2019 (COVID-19). Centers for Disease Control and Prevention. https://www.cdc.gov/coronavi ncov/hcp/guidance-risk-assesment-hcp.html. Published February 11, 2020. Accessed April 12, 2020.

23. Kunachak S, Sithisarn P, Kulapaditharom B. Are laryngeal papilloma virus-infected cells viable in the plume derived from a continuous mode carbon dioxide laser, and are they infectious? A preliminary report on one laser mode. J Laryngol Otol . 1996;110(11):1031-1033. doi:10.1017/s0022215100135686

24. Ferenczy A, Bergeron C, Richart RM. Human papillomavirus DNA in CO2 laser-generated plume of smoke and its consequences to the surgeon. Obstet Gynecol . 1990;75(1):114-118.

25. UPDATE: The Use of Personal Protective Equipment by Anesthesia Professionals during the COVID-19 Pandemic. https://www.asahq.org/about-asa/newsroom/news-releases/2020/03/update-the-use-of-personalprotective-equipment-by-anesthesia-professionals-during-the-covid-19-pandemic. Accessed April 7, 2020.

26. Liu Y, Yan L-M, Wan L, et al. Viral dynamics in mild and severe cases of COVID-19. Lancet Infect Dis . 2020;0(0). doi:10.1016/S1473-3099(20)30232-2

27. Yeh HC, Turner RS, Jones RK, Muggenburg BA, Lundgren DL, Smith JP. Characterization of Aerosols Produced during Surgical Procedures in Hospitals. Aerosol Sci Technol . 1995;22(2):151-161. doi:10.1080/02786829408959736

28. Wölfel R, Corman VM, Guggemos W, et al. Virological assessment of hospitalized patients with COVID2019. Nature . April 2020:1-10. doi:10.1038/s41586-020-2196-x

29. Pitkäranta A, Virolainen A, Jero J, Arruda E, Hayden FG. Detection of rhinovirus, respiratory syncytial virus, and coronavirus infections in acute otitis media by reverse transcriptase polymerase chain reaction. Pediatrics . 1998;102(2 Pt 1):291-295. doi:10.1542/peds.102.2.291

30. Hilal A, Walshe P, Gendy S, Knowles S, Burns H. Mastoidectomy and Trans-Corneal Viral Transmission: The Laryngoscope . 2005;115(10):1873-1876. doi:10.1097/01.mlg.0000177459.80574.2d

31. Workman AD, Welling DB, Carter BS, et al. Endonasal instrumentation and aerosolization risk in the era of COVID-19: simulation, literature review, and proposed mitigation strategies. Int Forum Allergy Rhinol . 2020;(Journal Article). doi:10.1002/alr.22577

\section{Hosted file}

HED-20-0585_COVID_table_v6.docx available at https://authorea.com/users/312328/articles/446108procedural-precautions-and-personal-protective-equipment-during-head-and-neck-instrumentationin-the-covid-19-era 